\title{
Numerical Analysis of Air Flow in a Modular Fan Unit Using CFD Simulation
}

\author{
Petr Zelenskýl ${ }^{1 *}$, Martin Barták ${ }^{1}$,Vojtěch Zavřel ${ }^{1}$, Vladimir Zmrhal ${ }^{1}$ and Radislav Krupa $^{2}$ \\ ${ }^{1}$ CTU Prague, University Centre for Energy Efficient Buildings, Czech Republic \\ ${ }^{2}$ ALTEKO 1td., Hostomice, Czech Republic
}

\begin{abstract}
The current regulation of European Commission No. 1253/2014 regarding Ecodesing requirements for ventilation units, which classified them based on the Specific Fan Power (SFP), has been driving the innovation in the sector of air-handling systems. The required energy saving can be met by applying modern and energy efficient Electronically Commutated (EC) motor which, at the same time, significantly change the internal geometry of the fan unit. The paper deals with design of a new type of modular fan unit ALTEKO Alton with EC motor and unhoused backward curved impeller. The design development and optimization of the fan unit leading to the reduction of generated aerodynamic noise, lower pressure loss and better outflow characteristics is supported by the Computational Fluid Dynamic (CFD) simulations, which enables visualization of air streamlines, velocities and pressure contours within the unit. Such a design is an iterative process between CFD specialist and construction team, where, based on the simulated results, the critical spots are identified, the geometry is improved and again verified throughout the CFD simulation. The paper describes in detail the individual steps of the optimization study, starting with the import of the detailed geometry from the design CAD software and its necessary simplification for the use in the CFD study, meshing of the model and consequent simulation. The methods of the fan rotation approximation are discussed as well. Additionally, the optimum position of heat exchanger in front of the fan unit is addressed. The presented work is a part of a project supported by the EU under the Operational Program Enterprise and Innovation for Competitiveness.
\end{abstract}

\section{Introduction}

The design approaches and tools in the field of Environmental Engineering are gradually changing with the increasing capabilities of the information technology. Standard design methods are complemented by other alternatives, such as simulations. The motivation for using simulation methods in the design process is to reduce the costs of design (development), manufacturing (implementation) and operation of equipment (or systems, buildings, etc.). The first important asset of simulations is their ability to provide otherwise inaccessible information that can assist during the design process, equipment sizing, etc. Another one is relatively easy availability of variant solutions, which can be used to optimize the proposed designs or operation settings. This can increase efficiency, quality, durability, safety, but also reduce the impact on the environment, both of new and existing equipment and systems.

This applies also to the computational fluid dynamics (CFD) modelling and simulation. It is increasingly used in design practice, as it can provide very detailed flow information about, i.e., pressure, velocity, temperature, turbulence intensity and other quantities in the solved domain. CFD technique allows the user to study the investigated domain at several levels of resolution at the same time. It provides both overall insight and detailed information about the flow and helps to better understand the hidden interactions. This ability is demonstrated below on the case study of the fan chamber of a modular air-conditioning unit. It is obvious that experimental assessment of detailed flow characteristics in such a complex geometry would be practically impossible, not to mention the unfeasibility of experimental variant studies of the chamber design.

Effective use of CFD simulations for practical applications depends on how difficult it is to create the numerical models, prepare the computational mesh and set up the simulation. There has been a significant development of software tools for pre-processing of models for CFD simulations in the recent years, which is partially described in this paper.

A two-year research project targeting optimization of fan chamber for modular air-conditioning units is taking place at CTU in Prague, in cooperation with ALTEKO ltd., an air conditioning systems manufacturer. A detailed CFD analysis of the air flow in the ALTEKO Tango 4 (currently produced) fan chamber was conducted during the first phase of the project in 2017. The noise parameters of the unit were determined experimentally. As the next step, a design of a new type of fan chamber ALTEKO Alton was proposed on the

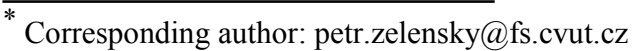


basis of the findings of the previous studies. The initial design was analysed using CFD simulations, during the second phase of the project in 2018, and optimized with use of the CFD analysis results. The prototype of the fan chamber Alton will be manufactured and noise parameters of the new unit will be determined experimentally.

The air flow through the fan chamber influences both the pressure losses of the air-conditioning unit (i.e. its energy efficiency) and the generated noise. The CFD analysis of the air flow provided background information for optimization of the internal geometry of the fan chamber in order to minimize local pressure losses and address the possible sources of aerodynamic noise. The goal of the project is an innovative design solution of the fan chamber with better energy efficiency and lower noise.

\section{Tools for preparation of numerical models for CFD simulations}

\subsection{Preparation of numerical model geometry}

Spatial (3D) geometry of the device to simulate can be obtained in two ways. The first method is to use documentation in the form of technical drawings or sketches. In such case, the numerical model must be created from the scratch in one of the numerous modelling software for CFD geometry preparation (for example in Design Modeller or SpaceClaim from ANSYS). This method has been widely used in the past, primarily due to the incompatibility of files created in CAD design software and CFD simulation software, causing that the models could not be easily transferred from one software tool to another. However, it can be time consuming and quite difficult to create the numerical model with complex geometries.

Currently, it is possible (and preferable) to import 3D geometry created by a designer in a CAD tool (such as Autodesk Inventor, SolidWorks, Catia, etc.) directly into the software tool for preparation of geometry for CFD simulation. It can be done in an easy way. Some of the universal file formats (such as STEP) can be used. There are also format converters implemented in the CFD geometry tools, which can be utilized. The advantage of this method is faster preparation of the geometry for CFD simulation and lower error rate in comparison to the previous method, when geometry is created from the scratch. However, it is always necessary to check the imported model and optimize the geometry for CFD simulation. Design models often contain imperfections that can be neglected in manufacturing documentation, but have to be addressed before CFD simulations. These may be for example missing surfaces on the geometrical bodies, minor collisions of individual parts, minor inaccuracies in their location, etc. Moreover, there are often geometric details that are insignificant for the solved CFD task but make the numerical mesh creation unnecessary difficult. Therefore, it is advisable to always simplify the geometry and remove undesirable details. Last but not least, it must be remembered that the subject of CFD simulation is often not the device itself, or its part, but the surrounding space filled with fluid. In such cases it is necessary to create an inverse model from the design geometry. From the ANSYS simulation software, all the described tasks can be dealt for example with SpaceClaim tool.

\subsection{Preparation of computational mesh}

CFD simulations are usually based on the finite volume method, i.e. division of the investigated domain into a large number of small control volumes and subsequent solution of conservation equations for mass, momentum, and energy for each of them. The quality of this numerical mesh significantly affects the convergence of the calculation, the quality and the accuracy of the obtained results. Therefore, it is necessary to pay close attention to its creation.

Historically, the basic elements of the numerical mesh are hexahedrons and tetrahedrons, see Fig. 1. The main disadvantage of hexagonal cells lies in their difficult application in domains with more complex geometries. The attempt to solve this issue and to automate the creation of numerical meshes has led to the use of tetrahedral cells that can more easily discretize geometrically complex areas. However, they have other drawbacks. Their volume is relatively small (for example, a regular tetrahedron has about eight times smaller volume than a hexahedron with the same edge length), so the number of cells in the computing area rises, resulting in higher demands on memory and computer power. Another problem with tetrahedral cells is tendency of the network generators to occasionally create high-skewed cells with very sharp angles. Their occurrence in the computational domain subsequently complicates the convergence of the calculation.

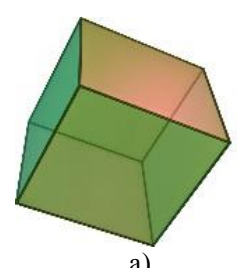

a)

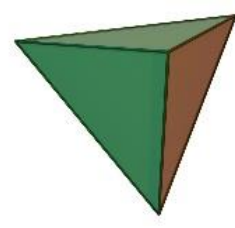

b)

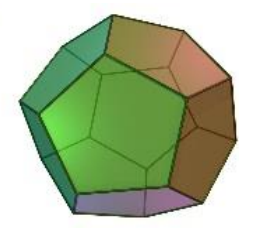

c)
Fig. 1. Basic elements of volume numerical mesh: a) hexahedron, b) tetrahedron, c) polyhedron [1]

The cells in the shape of polyhedrons (see Fig. 1), forming so-called polyhedral mesh, are a possible compromise. The number of cells of polyhedral mesh is significantly lower than that of the mesh with tetrahedral elements, while complex geometries can be discretized relatively easily.

Flow prediction in the near wall regions (so called boundary layer) with high gradients of velocity is crucial to the accuracy of CFD simulations of wall bounded turbulent flows which are often present in engineering applications. The presence of the solid wall significantly affects the transport of mass and momentum. In order to ensure correct calculation of flow characteristics near the walls, the polyhedral (or hexahedral, or tetrahedral) 
volume mesh in these regions can be replaced by multiple layers of prismatic cells, when each cell has a base of the corresponding surface mesh element.

From the ANSYS software, Fluent Meshing tool can be used to create polyhedral meshes with prismatic cell layers. Although it has relatively high demands on the user, it offers a robust semiautomatic mesh generator with a number of functions for subsequent modification and refinement of the created mesh.

\section{Numerical model and CFD simulation of Tango 4 fan chamber}

In the solved research project, the manufacturer provided a detailed 3D geometry of the Tango 4 fan chamber, see Fig. 2 - top. It was previously created in SolidWorks by fan chamber designer. The geometry was available in the universal format STEP and it could be imported directly into the ANSYS SpaceClaim as a spatial model.

The fan chamber has internal dimensions of $800 \mathrm{x}$ $500 \times 600 \mathrm{~mm}$ (width $\times$ height $\times$ length) and it covers the unhoused backward curved impeller mounted on a three phase induction motor attached to the supporting profiles at the bottom of the chamber.

After the quality control of the obtained geometry and correction of the minor deficiencies (correction of the axial alignment of the motor, impeller and fan inlet; correction of the colliding bodies' positions), the below listed simplifications were made. The modified geometry is shown in Fig. 2 - bottom. It was expected that all the simplification and geometry adjustments should have minimal influence on the flow in the fan chamber:

- nuts, washers, bolts and threaded rods were removed; all the holes after the removed parts were filled;

- technological fillets and cut-outs of sheet steel profiles were simplified;

- geometry of the profiles supporting the motor was simplified;

- geometry of the motor was simplified;

- geometry of the impeller was repaired, while preserving the main geometry of the blades and the flow channels (improperly divided surfaces were joined together, double edges and miniature surfaces were removed, technological fillets on the impeller blades were simplified, etc.);

- rectangular air ducts with lengths of $0.2 \mathrm{~m}$ and $0.3 \mathrm{~m}$ were created at the fan chamber inlet and outlet, respectively (not shown in Fig. 2).

The fan chamber geometry optimized for CFD simulation was imported into ANSYS Fluent Meshing. The volumetric model was automatically converted to a set of surfaces meshed by a triangular grid with predefined parameters, see Fig. 3. The surface mesh serves as the basis for the following volume discretization of the domain. Therefore, it must be sufficiently fine to capture all the important geometric details. It is also necessary to check its quality and, if necessary, repair problematic cells.
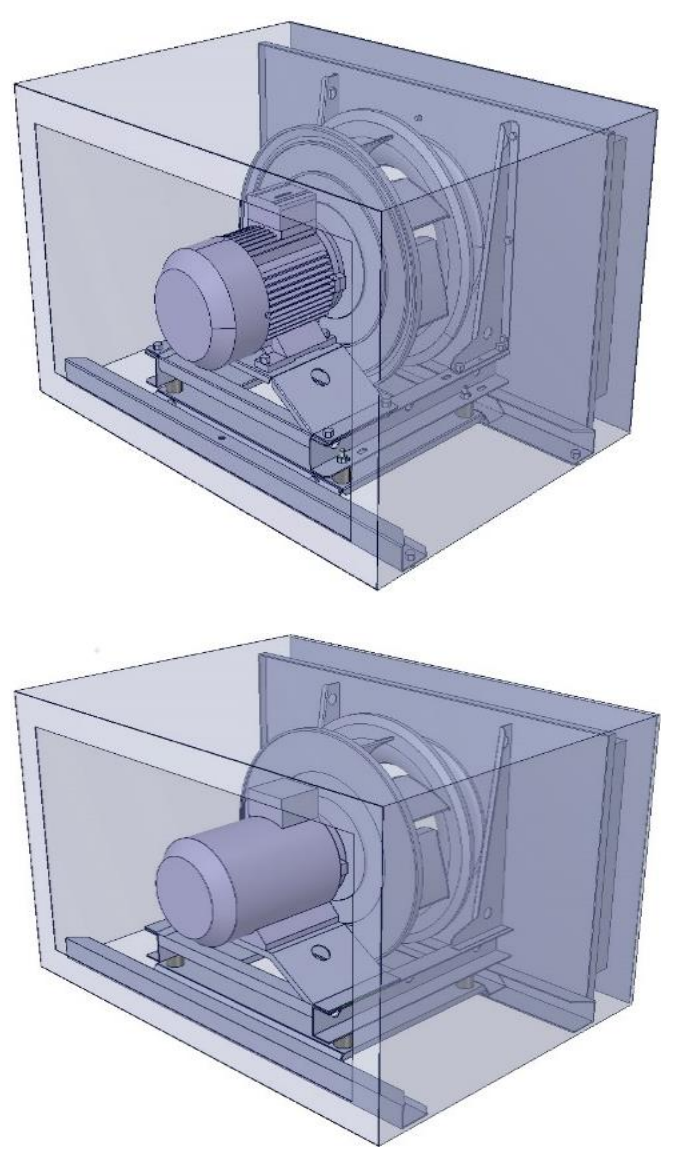

Fig. 2. Model of the fan chamber Tango 4; geometry from the CAD software (top) and geometry optimized for CFD (bottom)

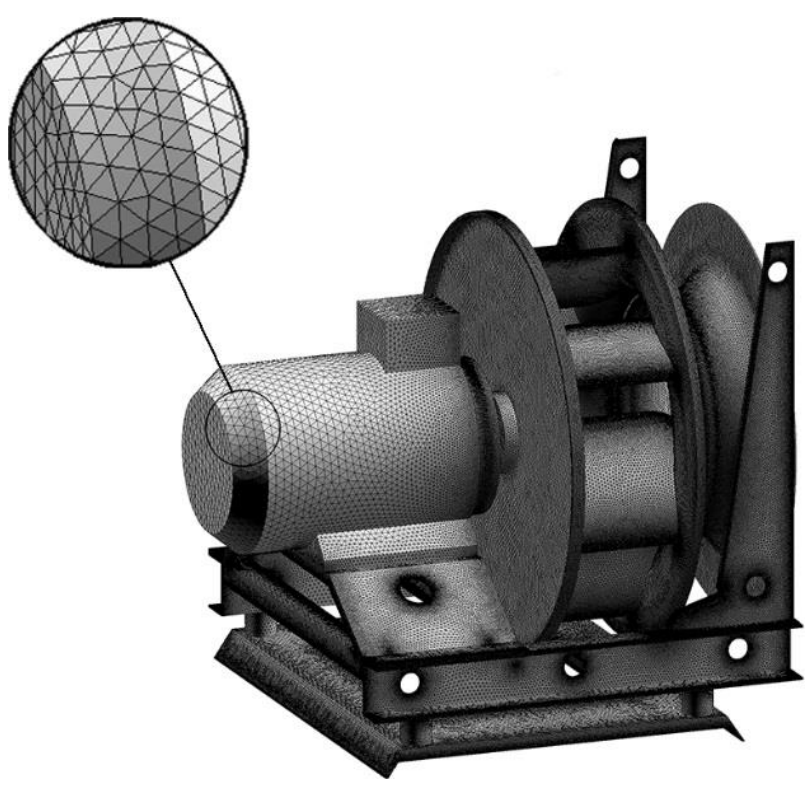

Fig. 3. Surface mesh on the motor and fan impeller

As the next step, a polyhedral volume grid with a prismatic boundary layer mesh on the impeller blades and surfaces of the fan inlet was created, see for example Fig. 4. The resulting mesh had 11.5 million computational cells in the basic variant and 34.8 million in a refined variant. 


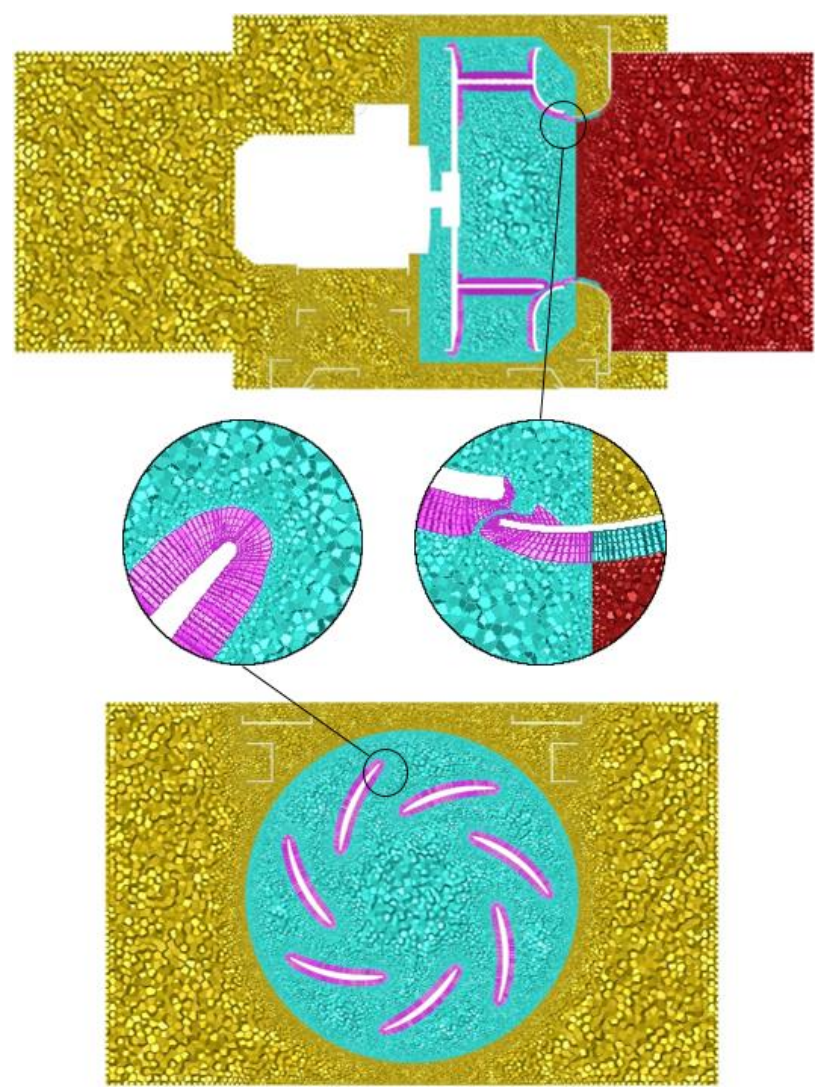

Fig. 4. Volume mesh of the model with prismatic boundary layer mesh on the selected surfaces - basic variant, $11.5 \mathrm{M}$ cells

\subsection{Numerical solution}

CFD simulations were solved using the software ANSYS Fluent 18.0. as a flow of incompressible air, without consideration of the energy equation. Five different models of turbulence were tested: three commonly used $k-\varepsilon$ models (model by Launder and Spalding [2], model by Yakhot and Orszag [3], and model by Shih et al. [4]), model $k$ - $\omega$ SST and Reynolds Stress Model (RSM). While the results of simulations with individual models of turbulence did not differ much from each other, the best convergence of the solution showed the two-equation model $k-\varepsilon$ according to Shih et al. [4] (so called $k-\varepsilon$ Realizable) with correction on the pressure gradient effects in the boundary layer. Therefore, this turbulence model was used for all the below presented calculation. The flow in the near-wallregions was calculated by integration of governing equations in the viscous sublayer. The PRESTO! scheme was chosen for the discretization of pressure equation as it is recommended for solving rotary machines [5]. The convective terms of the equation were solved using second order upwind scheme. Pressure and velocity fields were coupled by the SIMPLE algorithm.

Boundary condition at the inlet of the fan chamber was identical for all the simulations. The air was drawn from an open space, i.e. the static pressure at the inlet was $0 \mathrm{~Pa}$, intensity of turbulence was $0.1 \%$ and turbulent viscosity ratio 0.01 . The pressure outlet boundary condition was used at the exhaust from the fan chambers with an exhaust pressure corresponding to the simulated operating point of the fan, as specified below for individual simulations.

Correct approximation of the fan impeller rotation is the fundamental part of the CFD simulation of the fan chamber. There are two different methods available in ANSYS Fluent:

- Multiple Reference Frame (MRF) - the air flow is calculated in one single moment of the rotation, i.e. in one single impeller position (the method can also be referred to as the "frozen rotor approach"). It does not account for the relative motion of a moving zone with respect to adjacent zones. The impeller rotation is approximated by prescribing the tangential components of the velocity to the specified volume rotating at a chosen angular velocity. The result of the simulation is a stationary image of the flow in the domain.

- Sliding Mesh - the air flow is considered as unsteady. The rotation of the wheel is simulated directly, i.e. the relative motion of a moving zone (fan impeller) with respect to adjacent zones is considered and continuous change of the geometry occurs in the CFD simulation. The advantage of this method compared to the MRF approach is the potential higher accuracy of the obtained results. The disadvantage is considerably higher demand on computational time and difficult post-processing of the obtained results arising from the large number of data files obtained for individual positions of the fan impeller rotation.

\subsection{CFD results evaluation}

In the first step of the project, the rotation of the fan impeller was approximated by the MRF method. The rotating volume was closely encapsulating the impeller; see the cyan-coloured domain in Fig. 4. The simulation was done for four operating points of the fan at rotational speeds of 1996, 2126, 2284 and 2500 RPM and various total pressures for individual cases, see Table 1 .

Table 1. Pressure at the exhaust from the fan chamber Tango 4 (i.e. operating pressure of the fan)

\begin{tabular}{|c|c|}
\hline $\begin{array}{c}\text { Rotation speed } \\
{\left[\mathrm{min}^{-1}\right]}\end{array}$ & $\begin{array}{c}\text { Operating Pressure } \\
{[\mathrm{Pa}]}\end{array}$ \\
\hline 2500 & 680 \\
\hline 2284 & 640 \\
\hline 2126 & 600 \\
\hline 1996 & 580 \\
\hline
\end{tabular}

12,000 iterations were calculated for all the simulations. The residual values of the continuity equation were in the order of $10^{-5}$ at the end of the calculations, the residual values of the other solved equations were in the order of $10^{-6}$.

A very detailed analysis of air flow in the fan chamber can be carried out on the basis of the obtained results. See for example Fig. 5, which shows the velocity field in two perpendicular cross-sections of the fan chamber, or Fig. 6 showing the velocity vectors in the 
cross-section of the impeller, both at the rotation-speed of 2500 RPM.

In addition, the simulated volume flow rates of air at the exhaust of the fan chamber were compared with experimentally measured values for the four operating points of the fan. The experiment was conducted in the same manner as CFD simulations. The air was drawn directly from the room and the exhaust pressure was set according to the measured operating points of the fan, as specified in the Table 1.

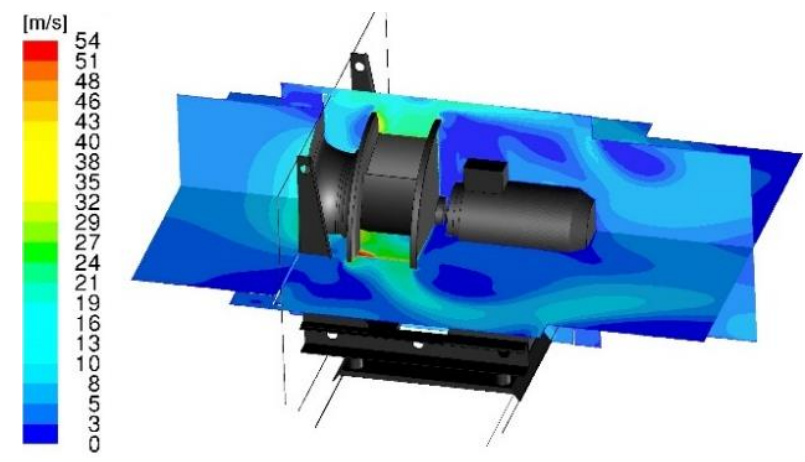

Fig. 5. Velocity field in the fan chamber

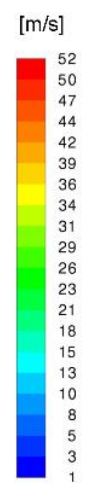

Fig. 6. Velocity vectors in cross-section of the impeller

The volume flow rates in the CFD simulations of the fan chamber using the MRF approach and the basic numerical mesh (11.5 million cells) were 16 to $24 \%$ lower in comparison to the measured values, see Table 2. Therefore, as the next step, the flow in the fan chamber was simulated using the Sliding Mesh method, which should provide better accuracy of the simulated results than the MRF method [6]. Due to the high computational demands of this approach, the simulation was solved only for one operating point of the fan with rotational speed 2500 RPM. The results of the simulation using the Sliding Mesh method were compared to the results of simulation with the MRF approach. The volume flow rates were similar in both cases, i.e. in the case of MRF approach $16 \%$ lower and in the case of Sliding Mesh approach $13 \%$ lower than the measured values at the given working pressure and rotational speed of the fan. Although the Sliding Mesh approach was closer to the experimental results, the MRF approach was used for further simulations as it has significantly lower computational demands and it is easier to evaluate the simulation results.

CFD simulation with the fine mesh (34.8 million cells) was performed for two operation points of the fan at rotational speeds 2126 and 2284 RPM. The purpose was to verify the independence of the simulation results on the density of the numerical mesh. The volume flow rates of the fan were lower than the measured values in both simulated cases, see Table 3 . The deviation was, similarly to the previous simulations with the basic mesh, in the range of 15 to $22 \%$.

Table 2. Volume flow rates - comparison of Tango 4 simulations with basic mesh ( $11.5 \mathrm{M}$ cells $)$ and measurement

\begin{tabular}{|c|c|c|c|}
\hline $\begin{array}{c}\text { Rotation speed } \\
{\left[\mathrm{min}^{-1}\right]}\end{array}$ & Measurement & \multicolumn{2}{|c|}{ Basic mesh } \\
\hline 2500 & $\left.4 \mathrm{~m}^{3} / \mathrm{h}\right]$ & {$\left[\mathrm{m}^{3} / \mathrm{h}\right]$} & {$[\%]$} \\
\hline 2284 & 3930 & 3708 & 84,2 \\
\hline 2126 & 3465 & 2988 & 76,0 \\
\hline 1996 & 2640 & 2664 & 76,9 \\
\hline
\end{tabular}

Table 3. Volume flow rates - comparison of Tango 4 simulations with refined mesh $(34.8 \mathrm{M}$ cells) and measurement

\begin{tabular}{|c|c|c|c|}
\hline $\begin{array}{c}\text { Rotation speed } \\
{\left[\mathrm{min}^{-1}\right]}\end{array}$ & Measurement & \multicolumn{2}{|c|}{ Refined mesh } \\
\hline 2284 & 3930 & 3356 & 85,4 \\
\hline 2126 & 3465 & 2707 & 78,1 \\
\hline
\end{tabular}

The basic numerical mesh was used for further work, due to the very high computational demands of the simulation with the refined numerical mesh (the size of the numerical domain and the number of cells was at the limit of the computational power of the information technology available at the Faculty of Mechanical Engineering CTU in Prague).

The cause of the lower air flow rates in the CFD simulations in comparison with the experimentally obtained data could be caused by the under prediction of the fan performance by the MRF approach, which has been previously discussed in the available literature $[6,7,8]$. This could have been addressed by increase of the fan rotation speed in CFD simulations, in comparison with the experiment, with the use of Fan Law [8]. This would have increased the volume flow rates through the simulated fan chamber. However, considering that the results of the CFD simulations were not meant for comparison with absolute, measured values, but they were used mainly to analyze the velocity field in the fan chamber and perform a variant study of different geometrical solutions (i.e. inter-model comparison), the deficiency of the CFD simulation results was considered as not significant for the solved research.

\section{Numerical model and CFD simulation of Alton fan chamber}

The second phase of the project, which was solved during the year 2018, focused on the design of a new type of fan chamber ALTEKO Alton. Same as the previous model Tango 4, it is a fan chamber with unhoused backward curved impeller. However, it is equipped with a new electronically commutated (EC) motor. 
The methodology established during the first phase of the project was used for the CFD simulations of Alton. Device geometry was acquired in STEP format and imported into the ANSYS SpaceClaim modeler as a spatial model. After checking its quality, the following adjustments were made (the optimized geometry for CFD simulation is displayed in Fig. 7):

- technological fillets and cut-outs of sheet steel profiles were simplified;

- geometry of the motor was simplified;

- the repaired geometry of the impeller was used - the geometry was prepared in the previous stage of the research (Tango 4), preserving the main geometry of the blades and flow channels;

- rectangular air ducts with lengths of $0.1 \mathrm{~m}$ and $0.5 \mathrm{~m}$ were created at the fan chamber inlet and outlet, respectively (not shown in Fig. 7).

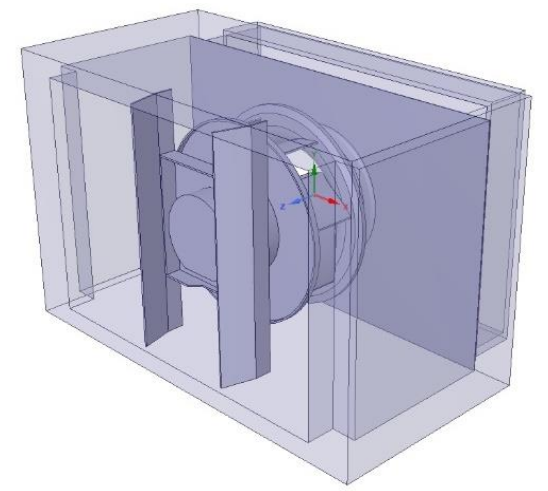

Fig. 7. Model of the fan chamber Alton optimized for CFD

The computational domain was meshed with a polyhedral grid with a prismatic boundary layer mesh on the impeller blades and surfaces of the fan inlet. Its parameters were based on the parameters of the basic mesh in the previous simulations of Tango 4 . The resulting volume mesh had 16.5 million computational cells.

The simulation setting was identically to the previous simulations of the Tango 4 fan chamber. The rotation of the fan impeller was approximated with use of the MRF method. The rotating volume was closely encapsulating the impeller. The calculation was done for three operating points of the fan at rotational speeds of 1996 , 2500 and 2970 RPM and various pressures at the fan chamber outlet (i.e. operating pressures), see Table 4.

Table 4. Pressure at the exhaust from the fan chamber Alton (i.e. operating pressure of the fan)

\begin{tabular}{|c|c|}
\hline $\begin{array}{c}\text { Rotation speed } \\
{\left[\mathrm{min}^{-1}\right]}\end{array}$ & $\begin{array}{c}\text { Operating Pressure } \\
{[\mathrm{Pa}]}\end{array}$ \\
\hline 2970 & 1300 \\
\hline 2500 & 800 \\
\hline 1996 & 580 \\
\hline
\end{tabular}

The results of simulation of air flow in the Alton fan chamber were compared to the experimentally obtained data, see Table 5. Similar to the simulation of Tango 4, the volume flow rates in the simulations of Alton fan chamber were approx. $25 \%$ lower than measured values.
Table 5. Volume flow rates - comparison of Alton CFD simulations and measurement

\begin{tabular}{|c|c|c|c|}
\hline $\begin{array}{c}\text { Rotation speed } \\
{\left[\mathrm{min}^{-1}\right]}\end{array}$ & $\begin{array}{c}\text { Measurement } \\
{\left[\mathrm{m}^{3} / \mathrm{h}\right]}\end{array}$ & \multicolumn{2}{c|}{ CFD Simulation } \\
{$\left[\mathrm{m}^{3} / \mathrm{h}\right]$} & {$[\%]$} \\
\hline 2970 & 4750 & 3348 & 70,5 \\
\hline 2500 & 4500 & 3456 & 76,8 \\
\hline 1996 & 3100 & 2394 & 77,2 \\
\hline
\end{tabular}

The velocity field in the fan chamber Alton was evaluated on the basis of the performed simulations in order to identify the possible imperfections of the proposed geometry (in terms of airflow deficiencies and generation of aerodynamic noise). Currently, the fan chamber geometry is being optimized, following the findings from the CFD simulations.

\section{Optimum position of heat exchanger}

Additionally, the optimum position of heat exchanger in front of the fan unit was studied. The uniformity of the air velocity field at the outlet from the heat exchanger was assessed, using uniformity index, for two types of heat exchangers (with depth of 44 and $176 \mathrm{~mm}$ ) and 5 different positions of heat exchanger in front of the fan chamber inlet. See Fig. 8 and Table 6.

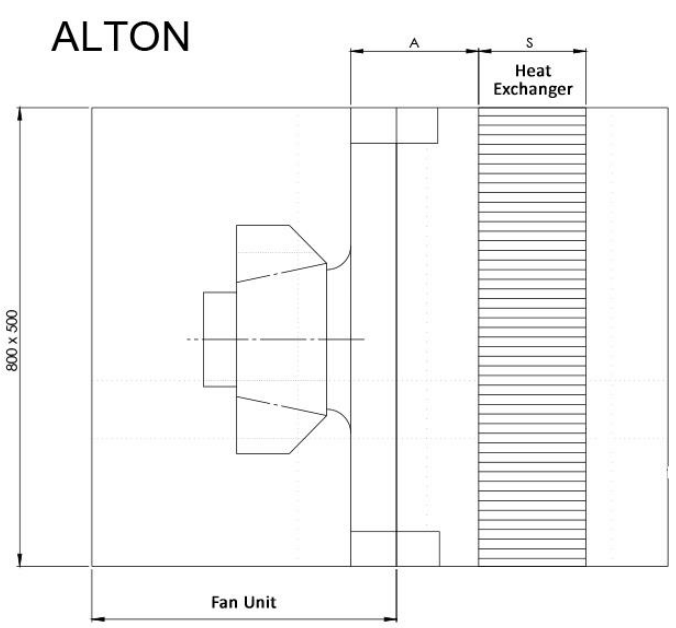

Fig. 8. Numerical study of heat exchanger optimum position

Table 6. Heat exchangers configurations

\begin{tabular}{|c|c|c|}
\hline Variant & A [mm] & S [mm] \\
\hline 1 & 140 & 44 \\
\hline 2 & 140 & 176 \\
\hline 3 & 170 & 44 \\
\hline 4 & 170 & 176 \\
\hline 5 & 200 & 44 \\
\hline 6 & 200 & 176 \\
\hline 7 & 230 & 44 \\
\hline 8 & 230 & 176 \\
\hline 9 & 260 & 44 \\
\hline 10 & 260 & 176 \\
\hline
\end{tabular}


The CFD simulations were performed according to the methodology established in the first phase of the research project (see Chapter 3). They were solved for the fan rotational speed of 2500 RPM. In order to lower the computational time of the simulations, only the heat exchanger chamber was simulated. The velocity field at the inlet to the fan chamber (outlet from the heat exchanger chamber) was defined from the previous simulations and prescribed as a boundary condition for fan total pressure $800 \mathrm{~Pa}$ (i.e. it was necessary to simulate the rotating fan impeller). The heat exchangers were modelled as a porous media and their pressure loss was calculated by the solver on the basis of the volume flow rate:

- exchanger $44 \mathrm{~mm}: \quad \Delta p_{z}=40 \cdot Q^{2}$

- exchanger $176 \mathrm{~mm}: \quad \Delta p_{z}=148 \cdot Q^{2}$

Velocity fields at the outlet from the heat exchangers are presented in Table 7 for all the simulated variants.

Table 7. Velocity field at the outlet from the heat exchangers

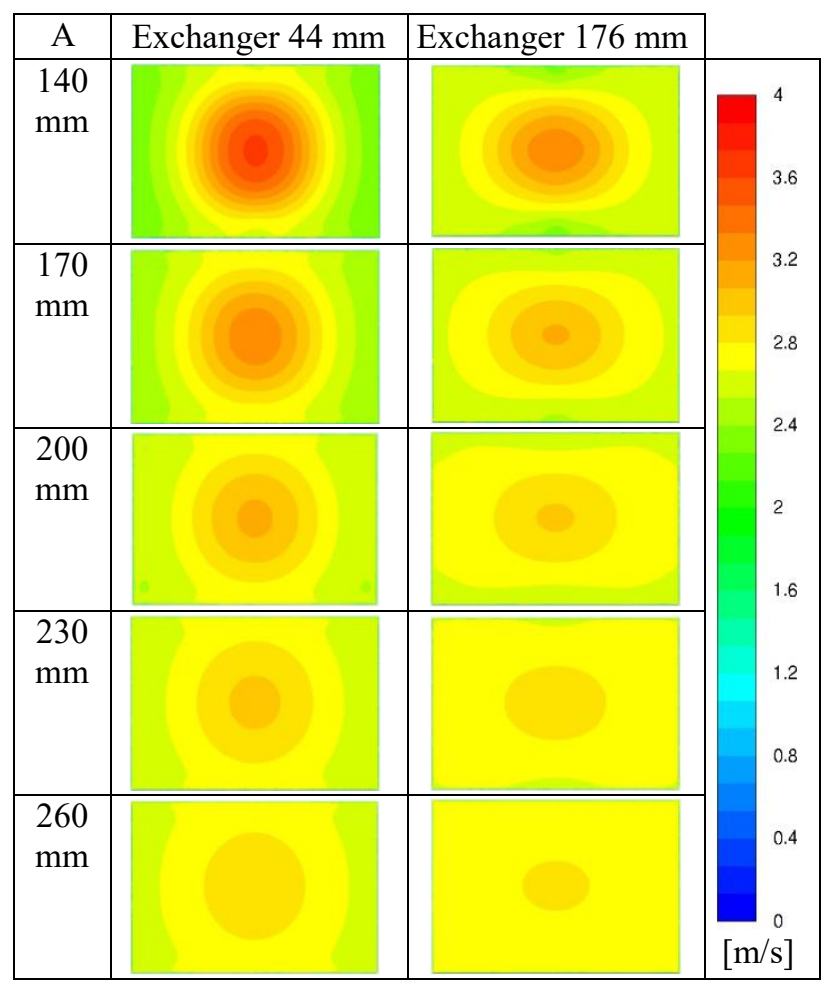

Aside of the velocity field at the heat exchanger outlet, the velocity profiles were evaluated as well. See for example Fig. 9 showing the horizontal velocity profile at the outlet of the heat exchanger $44 \mathrm{~mm}$, evaluated in the middle of the heat exchanger height (crossing the centre of the outlet surface).

Additionally, uniformity index of the velocity field at the heat exchanger outlet was evaluated, using the following formula:

$$
\gamma_{\mathrm{a}}=1-\frac{\sum_{i=1}^{n}\left[\left(\left|\phi_{i}-\bar{\phi}_{\mathrm{a}}\right|\right) A_{i}\right]}{2\left|\bar{\phi}_{\mathrm{a}}\right| \sum_{i=1}^{n} A_{i}}
$$

where $A_{i}$ is the area of the surface cell $i$ of the numerical grid (from the total number of cells on the outlet surface $n), \phi_{i}$ is the velocity in the given cell, and $\phi$ is the average velocity at the heat exchanger outlet. The results of the analysis are summarized in the Table 8 . The higher is the uniformity of the velocity field, the higher is the heat exchanger efficiency.

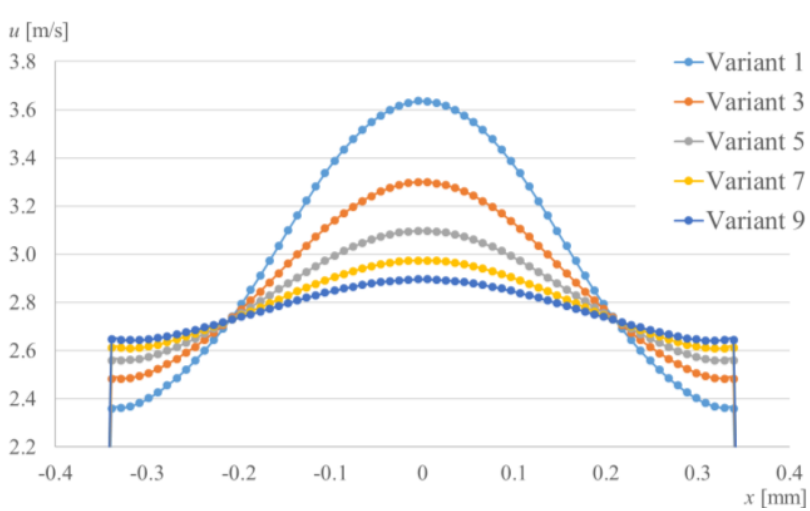

Fig. 9. Velocity profiles at the outlet from the heat exchanger $44 \mathrm{~mm}$, various positions in front of the fan chamber inlet

Table 8. Uniformity index of heat exchanger outlet for all the simulated variants

\begin{tabular}{|c|c|c|}
\hline $\mathrm{A}[\mathrm{mm}]$ & Exchanger $44 \mathrm{~mm}$ & Exchanger $177 \mathrm{~mm}$ \\
\hline 140 & $91.2 \%$ & $97.0 \%$ \\
\hline 170 & $94.4 \%$ & $98.1 \%$ \\
\hline 200 & $96.2 \%$ & $98.7 \%$ \\
\hline 230 & $97.4 \%$ & $99.1 \%$ \\
\hline 260 & $98.1 \%$ & $99.4 \%$ \\
\hline
\end{tabular}

The influence of the heat exchanger location on the air flow in front of the fan chamber is obvious from the presented results. The CFD simulations demonstrated the effect of two factors:

- the effect of the position of the exchanger in front of the fan chamber;

- the influence of heat exchanger depth (i.e. heat exchanger pressure loss).

In general, a more uniform velocity profile of the air flow through the heat exchanger, which increases the heat exchanger efficiency, can be achieved by using a larger depth exchanger (i.e. heat exchanger with higher pressure loss) and / or by placing the exchanger further away from the fan chamber inlet.

\section{Conclusion}

The aim of the paper was to inform the reader about the current methods of geometry preparation and numerical meshing for CFD simulation of equipment and systems in the field of Environmental Engineering. The individual steps of CFD simulation study were disused in detail, from the geometry preparation, through the creation of the numerical mesh, CFD simulation and result evaluation. Two approaches to account the influence of rotating parts of machines were discussed.

The use of CFD simulations for practical applications was demonstrated on the numerical variant study of air flow in two fan chambers for modular air conditioning 
units: ALTEKO Tango 4 (currently produced) and ALTEKO Alton (with innovated design).

The presented study was based on the geometry of the Tango 4 fan chamber created in SolidWorks by the manufacturer. The geometry was imported into ANSYS SpaceClaim and optimized for CFD simulation. It was converted to a triangular surface mesh and subsequently meshed by a polyhedral volumetric mesh with prismatic boundary layer. ANSYS Fluent Meshing was used to create the computational grids. The CFD simulation of the air flow in the fan chamber and the evaluation of the results were done in the ANSYS Fluent for four operation points of the fan, using two different approaches to approximate the influence of the rotational part of the device and two computational meshes with different density (basic mesh and refined mesh).

The airflow rates through the fan chamber in the CFD simulations were lower in comparison to the experimentally obtained values. This deficiency could have been caused by the under prediction of the fan performance by the MRF approach to approximate the propeller rotation, as discussed in the available literature. However, considering that aim of the CFD simulations (analysis of the velocity field in the fan chamber and variant study of different geometrical solutions, i.e. intermodel comparison), this deficiency was considered as not significant for the solved research and it was not studied in detail in the presented paper.

Additionally, the optimum position of heat exchanger in front of the fan unit was studied. The uniformity of the air velocity field at the outlet from the heat exchanger was assessed, using uniformity index, for two types of heat exchangers (with depth of 44 and $176 \mathrm{~mm}$ ) and 5 different positions of heat exchanger in front of the fan chamber inlet. The higher is the uniformity of the velocity field, the higher is the heat exchanger efficiency. In general, a more uniform velocity profile of the air flow through the heat exchanger can be achieved by using a larger depth exchanger (i.e. heat exchanger with higher pressure loss) and / or by placing the exchanger further away from the fan chamber inlet.

The presented results are part of the ongoing research project. The established methodology was used for CFD simulations of the initial design of the new ALTEKO Alton fan chamber, equipped with an EC motor. Currently, the fan chamber geometry is being optimized, following the findings from the CFD simulations. The target of the project is optimization of the initial design of the fan chamber and development of a new product of the ALTEKO ltd., manufacturer of modular air condition units, with improved internal air flow characteristics leading to reduction of generated aerodynamic noise, lower pressure loss and better outflow characteristics of the unit.

\section{Nomenclature}

$k$

$\left[\mathrm{m}^{2} / \mathrm{s}^{2}\right]$

$u \quad$ velocity

$Q \quad$ volume flow rate

$\Delta p_{z} \quad$ pressure loss

$\varepsilon \quad$ turbulent dissipation rate

$\omega$

specific dissipation rate

$[\mathrm{m} / \mathrm{s}]$

$\left[\mathrm{m}^{3} / \mathrm{s}\right]$

$[\mathrm{Pa}]$

$\left[\mathrm{m}^{2} / \mathrm{s}^{3}\right]$

$[1 / \mathrm{s}]$

\section{References}

1. Basic elements of numerical mesh: Wikimedia Commons.

2. B.E. Launder, D.B. Spalding, The numerical computation of turbulent flows. Computer Methods in Applied Mechanics and Energy, no. 3, pp. 269289, 1974.

3. V. Yakhot, S.A. Orszag, Renormalization group analysis of turbulence. Journal of Scientific Computing, vol. 1, pp. 3-51, 1986.

4. T. Shih, W. Liou, A. Shabbir, Z. Yang, J. Zhu, A new eddy viscosity model for high Reynolds number turbulent flows. Computers \& Fluids, vol. 24, pp. 227-238, 1995.

5. ANSYS Inc. Ansys Fluent Fluent User's Guide. USA: ANSYS Inc., 2013.

6. P. Gullberg, R. Sengupta, Axial Fan Performance Predictions in CFD, Comparison of MRF and Sliding Mesh with Experiments, In: Proceeding of the $11^{\text {th }}$ European Conference on Turbomachinery Fluid Dynamics and Thermodynamics, Istanbul, Turkey, 2011.

7. S. Moreau, M. Henner, J.F. Brouckaert, D. Neal, Numerical and Experimental Investigation of RotorStator Interaction in Automotive Engine Cooling Fan Systems, In: Proceeding of the $7^{\text {th }}$ European Conference on Turbomachinery Fluid Dynamics and Thermodynamics, Athens, Greece, 2007.

8. P. Gullberg, L. Löfdahl, S. Adelman, P. Nilsson, A correction method for stationary fan CFD MRF models, In: Proceeding of the SAE World Congress \& Exhibition, Detroit, USA, 2009

\section{Acknowledgement}

This study was funded by the European Union, the European Regional Development Fund, within the Operational Program Enterprise and Innovation for

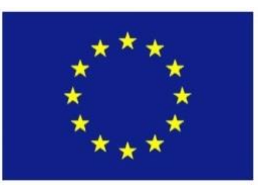
Competitiveness no. CZ01.1.02/0.0/0.0/15_019/0004577 